J. Phys. IV France 127 (2005) 69-75

(C) EDP Sciences, Les Ulis

DOI: $10.1051 / \mathrm{jp} 4: 2005127011$

\title{
Optiques multicouches pour l'extrême UV
}

\author{
F. Delmotte, J. Gautier, M.F. Ravet, F. Bridou et A. Jerome
}

Laboratoire Charles Fabry de I'Institut d'Optique, CNRS-UMR 8501, Bât. 503,

Centre Scientifique d'Orsay, 91403 Orsay Cedex, France

e-mail : franck.delmotte@iota.u-psud.fr

\begin{abstract}
Résumé. Nous présentons les principaux développements réalisés dernièrement en optique multicouches dans la gamme $30-50 \mathrm{~nm}$. Autour de $30 \mathrm{~nm}$, nous avons développé des multicouches à bande passante étroite dans le but de sélectionner une harmonique dans un spectre de source à génération d'harmonique d'ordre élevée. Dans cette même gamme spectrale, nous avons étudié des structures périodiques, à trois matériaux par période, qui permettent d'augmenter de manière significative la réflectivité. Enfin, pour les longueur d'onde plus importante (autour de $45 \mathrm{~nm}$ ) nous avons étudié les performances et la stabilité de multicouches à base de scandium : $\mathrm{Si} / \mathrm{Sc}$ et $\mathrm{Si} / \mathrm{X} / \mathrm{Sc} / \mathrm{X}$ ou $\mathrm{X}$ représente une couche barrière.
\end{abstract}

\section{INTRODUCTION}

Les miroirs interférentiels multicouches, dont le principe est basé sur le dépôt alterné de couches minces (de 1 à $20 \mathrm{~nm}$ d'épaisseur), présentent aujourd'hui un intérêt tout particulier lié d'une part au développement de la lithographie extrême UV et des nouvelles sources de rayons X (rayonnement synchrotron, plasmas créés par laser de puissance, lasers à rayons $\mathrm{X}$, génération d'harmoniques) et d'autre part, au besoin en diagnostics de plasmas chauds (observation du soleil ou des plasmas créés par lasers de puissance).

Depuis plus de 20 ans, le Laboratoire Charles Fabry de l'Institut d'Optique (LCFIO) est impliqué dans la conception, la fabrication et l'étude de systèmes optiques multicouche X-UV, principalement pour des applications liées à l'astronomie et au diagnostic de plasma. Depuis 1999, le LCFIO a créé, avec d'autres laboratoires d'Orsay impliqués dans cette thématique, le Pôle d'optique des Rayons X d'Orsay (PRaXO). Ce pôle a notamment œuvré pour la création de la Centrale d'Elaboration et de Métrologie d'Optiques X (nommée CEMOX), constitué d'un système de dépôt de multicouches opérationnel depuis novembre 2002 et d'une station de calibration (réflectomètre EUV avec source laser plasma) en cours de développement.

Actuellement, notre recherche est essentiellement axée sur la gamme spectrale 13-60 nm, gamme dans laquelle nous développons et étudions divers systèmes multicouches. Les multicouches $\mathrm{Mo} / \mathrm{Si}$ représentent le système de référence dans la gamme 13-30 nm, notamment pour la lithographie EUV à 13,4 nm. Des multicouches de $\mathrm{Mo} / \mathrm{Si}$ ont été optimisées et déposées dernièrement au LCFIO sur les miroirs des télescopes EUVI/SECCHI de la mission STEREO. Il s'agit de télescopes imageurs EUV à 4 canaux compris entre $17,1 \mathrm{~nm}$ et $30,4 \mathrm{~nm}$. [1]

Nous décrivons ici les principaux résultats obtenus dans la gamme $30-50 \mathrm{~nm}$. Après une brève description du dispositif expérimental, nous aborderons les trois thèmes suivants. L'étude et le développement de multicouches à base d'éléments "légers », tels que le carbure de bore $\left(\mathrm{B}_{4} \mathrm{C}\right)$ ou le nitrure de silicium $\left(\mathrm{SiN}_{\mathrm{x}}\right)$, a permis d'obtenir des miroirs à bande passante étroite tout en conservant une bonne réflectivité. Nous montrerons ensuite comment les multicouches à trois matériaux par période permettent d'améliorer les performances des miroirs en terme de réflectivité. Enfin, nous présenterons une étude des performances et de la stabilité de multicouches à base de scandium. 


\section{DISPOSITIF EXPÉRIMENTAL}

Un réacteur de dépôt par pulvérisation cathodique magnétron, dédié à la réalisation de multicouches pour l'optique X-UV, a été implanté dans la salle blanche du LCFIO en novembre 2002. Ce réacteur, entièrement automatisé, permet de concevoir et de réaliser de nouveaux systèmes multicouches et notamment des systèmes multimatériaux (3 ou 4 matériaux par période).

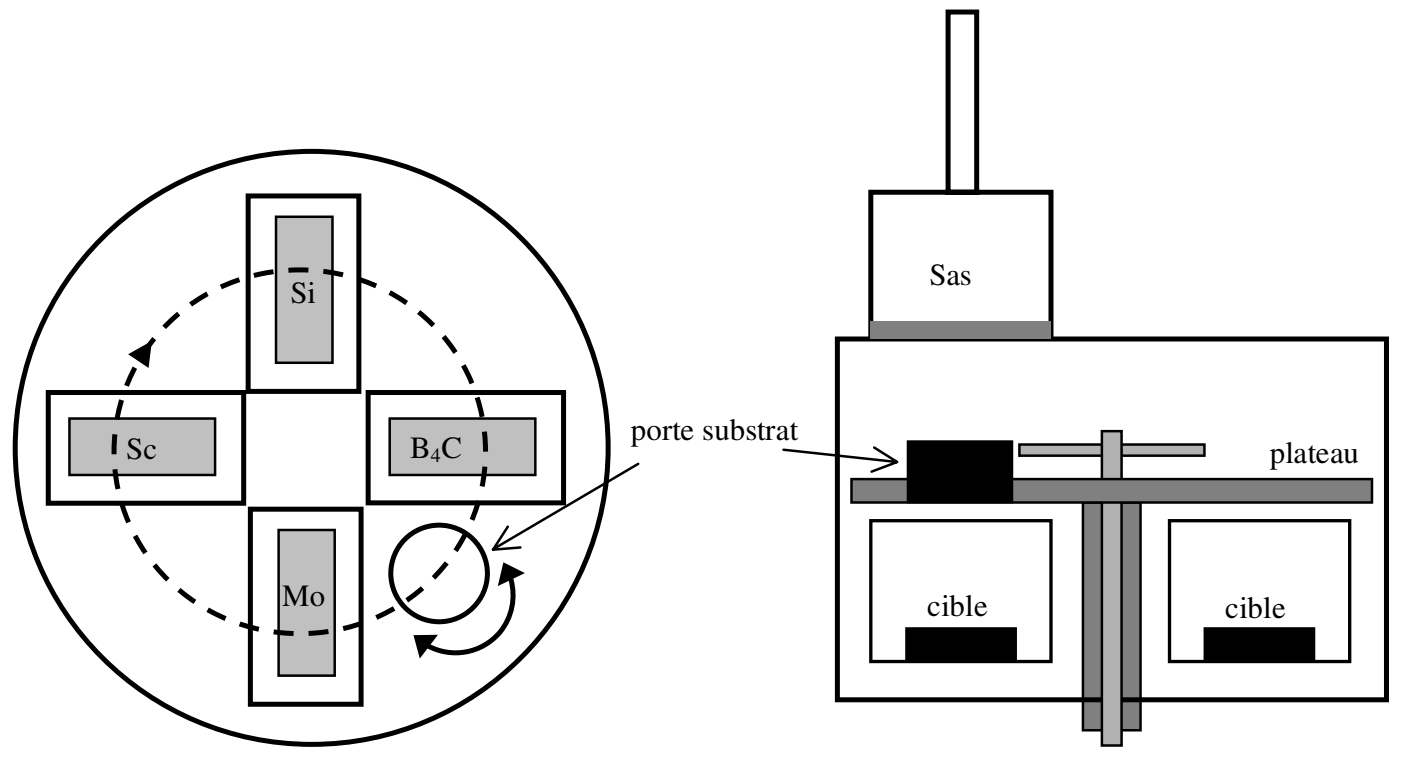

Figure 1. Schéma du réacteur de pulvérisation cathodique magnétron.

Le schéma du réacteur est représenté sur la figure 1 . Quatre cibles de dimension $80 \times 200 \mathrm{~mm}^{2}$ sont installées dans le bâti sous un vide de l'ordre de $2 \times 10^{-8}$ Torr : Si, B 4 C, Mo et Sc. De plus, des arrivées de gaz réactif $\left(\mathrm{N}_{2}\right.$ et $\left.\mathrm{O}_{2}\right)$ permettent de réaliser des nitrures ou oxydes de ces matériaux. Par exemple, le nitrure de silicium $\left(\mathrm{SiN}_{\mathrm{x}}\right)$ est déposé par pulvérisation réactif de la cible de silicium avec un mélange de gaz argon et azote. Un sas d'introduction permet de charger des échantillons jusqu'à 120 mm de diamètre dans la chambre de dépôt. Les dépôts de films minces ou de multicouches sont réalisés en mode dynamique : l'échantillon est satellisé et passe successivement au dessus des cibles souhaitées. La vitesse de passage et la puissance (RF ou DC) appliquée à la cible permettent de contrôler l'épaisseur de dépôt à $0,1 \mathrm{~nm}$ près.

\section{MIROIRS MULTICOUCHES À BANDE PASSANTE ÉTROITE}

Des multicouches à base d'éléments « légers », telles que $\mathrm{B}_{4} \mathrm{C} / \mathrm{Si}$ ou $\mathrm{SiN}_{\mathrm{x}} / \mathrm{Si}$, on été étudiées pour obtenir des miroirs à bande passante étroite tout en conservant une bonne réflectivité pour des longueurs d'onde voisines de $32 \mathrm{~nm}$. Le domaine d'application de ces multicouches concerne la sélection d'une harmonique dans un spectre de source à génération d'harmonique d'ordre élevé. L'objectif est de réfléchir au mieux une harmonique et d'atténuer les harmoniques voisines. Nous présentons sur la figure 2, les spectres de réflectivité en fonction de la longueur d'onde calculés pour des multicouches $\mathrm{SiN}_{\mathrm{x}} / \mathrm{Si}, \mathrm{B} 4 \mathrm{C} / \mathrm{Si}$ et $\mathrm{Mo} / \mathrm{Si}$ en incidence normale. Pour chaque multicouche, le pic de réflectivité est centrée sur l'harmonique 25 (à $32 \mathrm{~nm}$ ), le nombre de période est 100 et l'épaisseur de silicium représente $80 \%$ de l'épaisseur de la période. Les harmoniques voisines $\mathrm{H} 23$ et H27, respectivement à 34,8 nm et 29,6 nm, sont représentées par des traits en pointillé sur la figure. Ces simulations montrent clairement que les multicouches $\mathrm{Mo} / \mathrm{Si}$ classiquement utilisés dans cette gamme de longueur d'onde ne sont pas efficaces pour l'application 


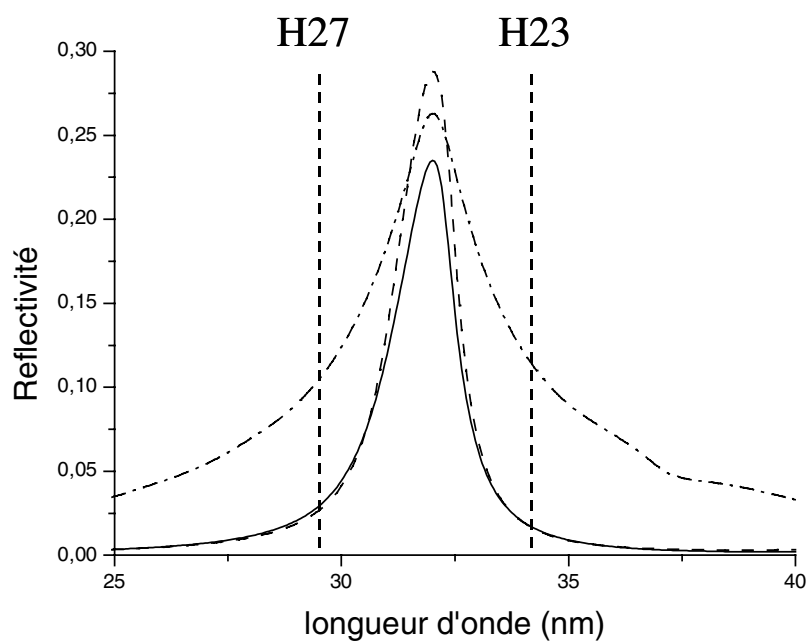

Figure 2. Réflectivité simulée en incidence normale des multicouches $\mathrm{B}_{4} \mathrm{C} / \mathrm{Si}$ (tirets), $\mathrm{SiN}_{\mathrm{x}} / \mathrm{Si}$ (trait continu) et $\mathrm{Mo} / \mathrm{Si}$ (tiret-point).

visée. D'où l'intérêt de développer des miroirs multicouches tels que $\mathrm{B}_{4} \mathrm{C} / \mathrm{Si}$ ou $\mathrm{SiN}_{\mathrm{x}} / \mathrm{Si}$, qui présentent théoriquement des bandes passantes plus étroites en conservant une réflectivité acceptable.

Expérimentalement, des multicouches $\mathrm{SiN}_{\mathrm{x}} / \mathrm{Si}$ avec 20 périodes ont été réalisées avec différentes conditions de dépôt. Les taux de réjection de harmoniques $\mathrm{H} 23$ et $\mathrm{H} 27$ ont été estimés respectivement de l'ordre de 10 et 8 , ce qui est tout a fait acceptable pour l'application visée. Par contre, la réflectivité maximale obtenue à $32 \mathrm{~nm}$ avec ce système reste très inférieure à la réflectivité théorique : elle est comprise entre $10 \%$ et $13 \%$ suivant les conditions de dépôt.

Des multicouches $\mathrm{B}_{4} \mathrm{C} / \mathrm{Si}$ avec 20 périodes optimisées pour sélectionner l'harmonique 25 à $32 \mathrm{~nm}$ ont également été réalisées. A partir des bandes passantes mesurées sous $10^{\circ}$ d'incidence, on peut estimer des taux de réjection des harmoniques 23 et 27 de l'ordre de 10 (3 à 4 fois supérieurs à ceux obtenus avec une multicouche $\mathrm{Mo} / \mathrm{Si}$ ). La réflectivité maximale pour le système $\mathrm{B}_{4} \mathrm{C} / \mathrm{Si}$, comprise entre $22 \%$ et $25 \%$ suivant les conditions de dépôt, est semblable à celle obtenue avec des multicouches $\mathrm{Mo} / \mathrm{Si}$ à cette longueur d'onde [2]. Un spectre typique d'une multicouche $\mathrm{B}_{4} \mathrm{C} / \mathrm{Si}$ mesurée sur rayonnement synchrotron sous $10^{\circ}$ d'incidence est présenté sur la figure 3. En conclusion, les multicouches $\mathrm{B}_{4} \mathrm{C} / \mathrm{Si}$

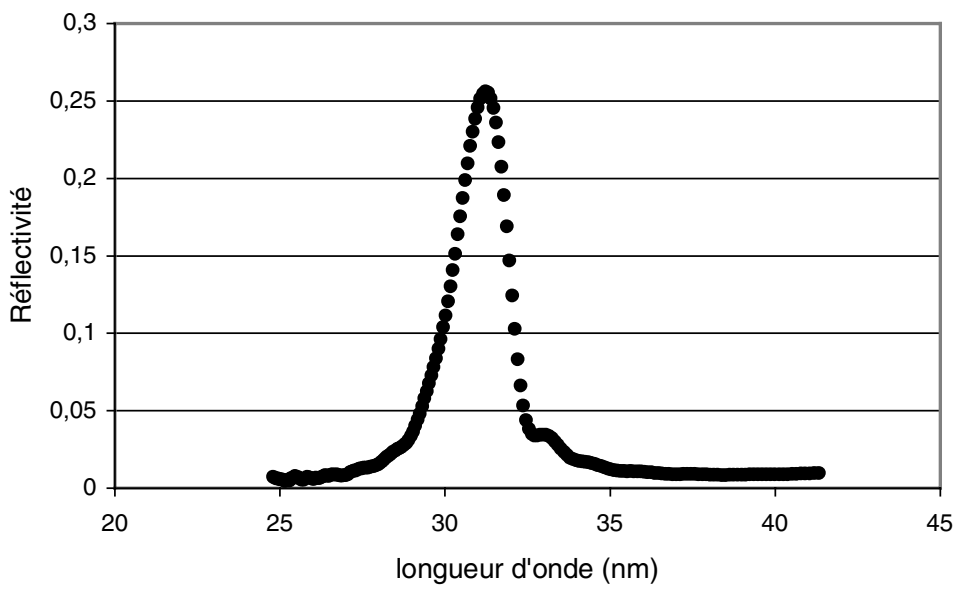

Figure 3. Réflectivité mesurée d'une multicouche $\mathrm{B}_{4} \mathrm{C} / \mathrm{Si}$ sous $10^{\circ}$ d'incidence en polarisation $\mathrm{S}$. 
développées au cours de cette étude répondent aux spécifications de l'application visée et seront utilisées prochainement dans la réalisation d'un intéféromètre XUV à source à génération d'harmonique développé en collaboration avec le CEA de Saclay.

\section{MULTICOUCHES À TROIS MATÉRIAUX PAR PÉRIODE}

Les systèmes multicouches classiques (à deux matériaux par période) qui sont utilisés autour de 30 $\mathrm{nm}$ présentent des réflectivité maximale de l'ordre de $25 \%$ [1, 3]. Ces réflectivités restent relativement faibles comparées à ce qui est atteint expérimentalement à d'autres longueurs d'onde (notamment entre $13 \mathrm{~nm}$ et $20 \mathrm{~nm}$ ). On peut se demander si des structures plus complexes permettraient d'atteindre des réflectivités plus élevées autour de $30 \mathrm{~nm}$. J. Larruquert a étudié théoriquement des structures quasipériodiques constituées de plus de deux matériaux et a montré par des simulations qu'il est possible d'obtenir des réflectivités plus élevées avec de telles structures (plus de 50\% en théorie avec 3 matériaux) [4]. Cependant, du point de vu expérimental, de telles structures sont très difficile à réaliser et surtout à contrôler du fait du nombre de matériaux présents et de la non périodicité de la structure. Nous nous sommes donc intéressés à des systèmes périodiques constitués de 3 matériaux par période, dans le but de vérifier théoriquement et expérimentalement la possibilité d'atteindre des réflectivités plus élevées autour de $30 \mathrm{~nm}$ avec de telles structures.

Nous avons simulé la réflectivité de multicouches à trois matériaux par périodes constitués de Mo et/ou Si et/ou $\mathrm{SiN}_{\mathrm{x}}$ et/ou $\mathrm{B}_{4} \mathrm{C}$. Pour chaque triplet de matériaux nous avons étudié l'effet de l'ordre des matériaux et l'effet de la nature du matériau qui termine l'empilement (matériau en surface). Pour chaque multicouche, les épaisseurs des trois matériaux ont été optimisées de manière à obtenir une réflectivité maximale à $32 \mathrm{~nm}$. Pour les quatre triplets étudiés, l'ajout d'un troisième matériaux dans la période permet d'améliorer la réflectivité si l'ordre des matériaux est bien choisi. La réflectivité optimale à $32 \mathrm{~nm}$ est obtenue avec une multicouche de type $\mathrm{B}_{4} \mathrm{C} / \mathrm{Mo} / \mathrm{Si}$. Les épaisseurs optimisées de ces trois matériaux sont respectivement $4,1 \mathrm{~nm}, 2,2 \mathrm{~nm}$ et $11,3 \mathrm{~nm}$. La réflectivité théorique est de $41 \%$ alors qu'elle n'est que de $34 \%$ pour une multicouche classique optimale de type $\mathrm{B}_{4} \mathrm{C} / \mathrm{Si}$.

Expérimentalement, plusieurs multicouches $\mathrm{B}_{4} \mathrm{C} / \mathrm{Mo} / \mathrm{Si}$ ont été réalisées et mesurées sur rayonnement synchrotron. La réflectivité obtenue est de l'ordre de $34 \%$, ce qui représente une augmentation importante par rapport à une multicouche classique de type $\mathrm{B}_{4} \mathrm{C} / \mathrm{Si}$. La figure 4 présente le spectre



Figure 4. Réflectivité mesurée à $10^{\circ}$ d'incidence sur une multicouche $\mathrm{B} 4 \mathrm{C} / \mathrm{Mo} / \mathrm{Si}$ (points) et réflectivité simulée à partir des paramètres déduits de réflectométrie X-rasant à $0,154 \mathrm{~nm}$ (trait). Voir le texte pour le détail. 
de réflectivité mesuré d'une multicouche $\mathrm{B}_{4} \mathrm{C} / \mathrm{Mo} / \mathrm{Si}$ de 15 périodes optimisée à $32 \mathrm{~nm}$. Cette multicouche a également été caractérisée par réflectométrie de rayons $\mathrm{X}$ rasant à $0,154 \mathrm{~nm}$. Par ajustement de la courbe expérimentale, on peut déduire les paramètres de cette multicouche [5] : les épaisseurs de $\mathrm{B}_{4} \mathrm{C}$, Mo et $\mathrm{Si}$ sont respectivement 4,5 nm, 2,7 nm et 11,2 nm et les rugosités des interfaces sont comprises entre 0,4 et $0,6 \mathrm{~nm}$. Ces paramètres ont été utilisés pour simuler le spectre de réflectivité de la multicouche autour de $32 \mathrm{~nm}$, en utilisant les indices tabulés des matériaux. Cette courbe simulée est représentée par un trait sur la figure 4 pour comparaison. Ces résultats montrent que le système à 3 matériaux par période $\mathrm{B}_{4} \mathrm{C} / \mathrm{Mo} / \mathrm{Si}$ permet d'atteindre des réflectivités élevées autour de $32 \mathrm{~nm}$. Des études sont en cours pour étendre ces résultats à d'autres longueurs d'onde.

\section{MULTICOUCHES À BASE DE SCANDIUM}

Nous avons déposé et étudié des multicouches à base de scandium dans le but de réaliser des miroirs pour des longueurs d'onde voisines de $45 \mathrm{~nm}$. Des études antérieures ont montré l'intérêt des multicouches $\mathrm{Sc} / \mathrm{Si}$ pour ces longueurs d'onde et ont souligné les problèmes de stabilité thermique de ce type de structure [6-8]. Les deux solutions proposées dans la littérature pour améliorer cette stabilité est l'ajout de W [7] ou de Cr [8] aux deux interfaces Sc sur Si et Si sur Sc. Cependant, l'ajout de ces couches barrières se fait au détriment de la réflectivité.

Le but de notre étude est de développer une structure de type $\mathrm{Si} / \mathrm{X} / \mathrm{Sc} / \mathrm{X}$, où $\mathrm{X}$ représente la couche barrière permettant d'améliorer la stabilité thermique de la structure en conservant la réflectivité initiale de la multicouche $\mathrm{Si} / \mathrm{Sc}$. Pour cela nous avons comparé les réflectivités obtenues avec et sans couche barrière, avant et après recuit thermique. Les matériaux que nous avons utilisés comme couche barrières sont $\mathrm{B}_{4} \mathrm{C}$, $\mathrm{SiN}_{\mathrm{x}}, \mathrm{ScN}_{\mathrm{x}}$. L'épaisseur visée pour les couches barrières est de $0,5 \mathrm{~nm}$ dans les trois cas. Nous avons réalisés différentes multicouches $\mathrm{Si} / \mathrm{Sc}$ et $\mathrm{Si} / \mathrm{X} / \mathrm{Sc} / \mathrm{X}$ de 15 périodes chacune. Dans chaque cas, l'épaisseur visée pour la période était de 25,6 nm et le dernier matériau déposé était du silicium. Des recuits thermiques ont été réalisés sur ces multicouches à l'aide d'un four à recuit rapide, à $200^{\circ} \mathrm{C}$ pendant 1 minute sous air. Les réflectivités mesurées avant et après recuit thermique sont représentés sur la figure 5 pour les quatre structures.

Comme l'indique la figure 5(a), la multicouche $\mathrm{Sc} / \mathrm{Si}$ non recuite présente une réflectivité de $35 \%$ à $46,2 \mathrm{~nm}$. Le recuit a pour effet de réduire la réflectivité de 1,5\% et de décaler le pic de réflectivité vers les basses longueurs d'onde de $\Delta \lambda=0,9 \mathrm{~nm}$ par contraction de la période. L'ajout de $\mathrm{B}_{4} \mathrm{C}$ aux interfaces (figure 5(b)) n'affecte presque pas la réflectivité de la structure (on conserve plus de $33 \%$ avant recuit) et limite la contraction de période après recuit (le pic ne se décale plus que de $\Delta \lambda=0,3 \mathrm{~nm}$ ). La réflectivité après recuit chute toujours de 1,5\%. Les deux autres barrières étudiées $\left(\mathrm{SiN}_{\mathrm{x}}\right.$ et $\left.\mathrm{ScN}_{\mathrm{x}}\right)$ sont plus efficaces en se qui concerne la stabilité après recuit : sur les figures 5(c) et 5(d), les réflectivités mesurées avant et après recuit sont identiques et le décalage du pic est négligeable. Le $\mathrm{ScN}_{\mathrm{x}}$ semble toutefois préférable au $\mathrm{SiN}_{\mathrm{x}}$ car il permet de conserver une meilleure réflectivité avant recuit (de l'ordre de $30 \%$ contre $26 \%$ pour le $\mathrm{SiN}_{\mathrm{x}}$ ).

Nous pouvons donc conclure que le système $\mathrm{Si} / \mathrm{B}_{4} \mathrm{C} / \mathrm{Sc} / \mathrm{B}_{4} \mathrm{C}$ présente une réflectivité optimale et permet de limiter la contraction de période qui apparaît dans le système $\mathrm{Sc} / \mathrm{Si}$. Le système $\mathrm{Si} / \mathrm{ScN}_{\mathrm{x}} / \mathrm{Sc} / \mathrm{ScN}_{\mathrm{x}}$ présente quant à lui une stabilité thermique optimale et un pouvoir réflecteur de l'ordre de $30 \%$ pour les longueurs d'onde voisines de $45 \mathrm{~nm}$.

\section{CONCLUSION}

Les optiques multicouches que nous avons développées ouvrent de nouvelles perspectives d'application dans la gamme de longueur d'onde 30-50 nm. L'étude et le développement de multicouches $\mathrm{B}_{4} \mathrm{C} / \mathrm{Si}$ a permis d'obtenir des miroirs à bande passante étroite tout en conservant une bonne réflectivité autour de $32 \mathrm{~nm}$ (plus de 25\%). Ces multicouches présentent un intérêt tout particulier pour le filtrage des 

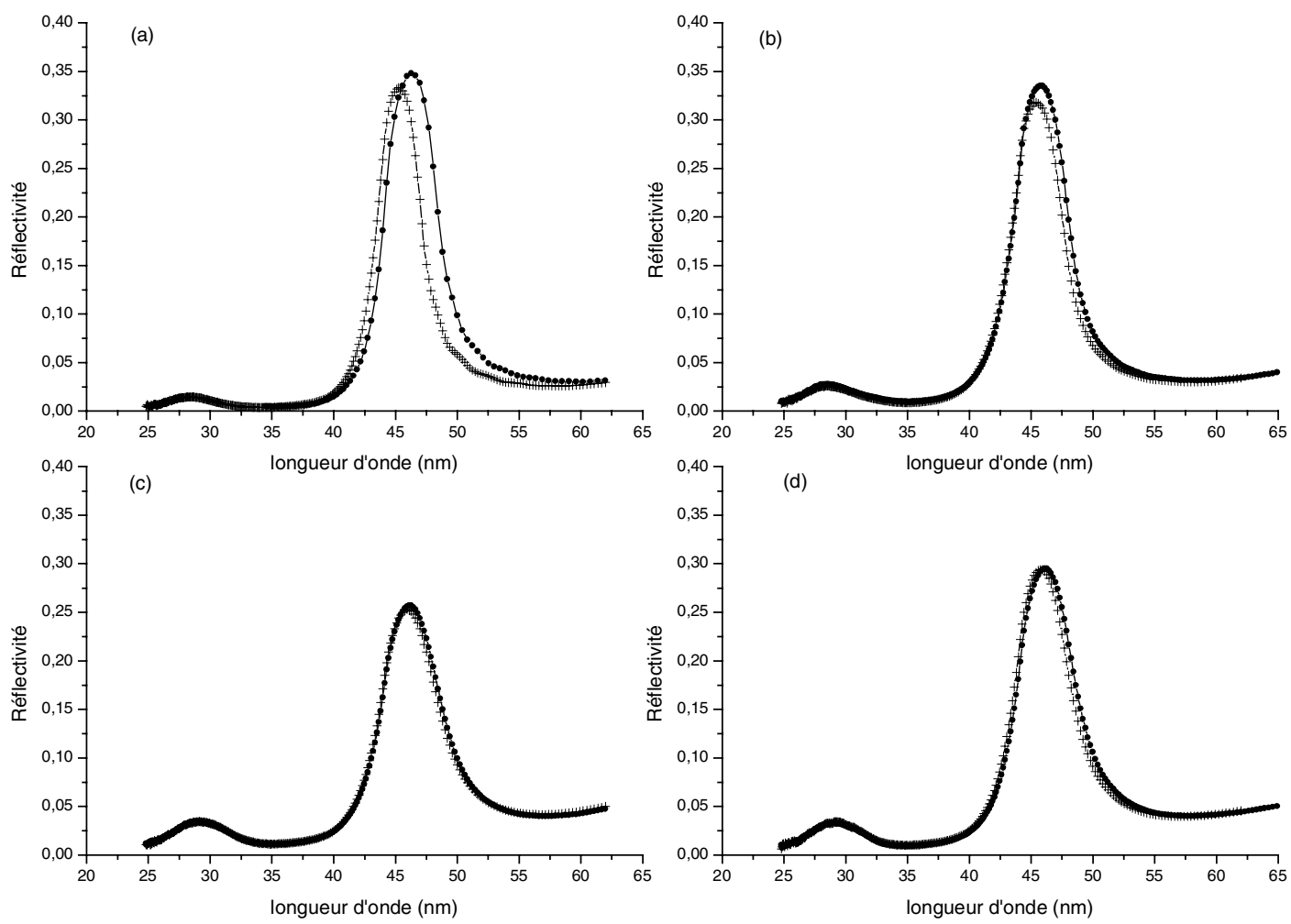

Figure 5. Réflectivité mesurée à $10^{\circ}$ d'incidence sur des multicouches (a) $\mathrm{Si} / \mathrm{Sc}$, (b) $\mathrm{Si} / \mathrm{B}_{4} \mathrm{C} / \mathrm{Sc} / \mathrm{B}_{4} \mathrm{C}$, (c) $\mathrm{Si} / \mathrm{SiN}_{\mathrm{x}} / \mathrm{Sc} / \mathrm{SiN}_{\mathrm{x}}$ et (d) $\mathrm{Si} / \mathrm{ScN}_{\mathrm{x}} / \mathrm{Sc} / \mathrm{ScN}_{\mathrm{x}}$. Les points représentent les mesures avant recuit thermique et les croix les mesures après recuit thermique (voir le texte pour les détails).

sources à génération d'harmoniques d'ordre élevé. Nous avons également montré que la réflectivité des optiques multicouches autour de $32 \mathrm{~nm}$ pouvait être améliorée de manière significative par la réalisation de structures périodiques à trois matériaux par période : un pouvoir réflecteur de $34 \%$ à $32 \mathrm{~nm}$ a été mesuré pour une multicouche $\mathrm{B}_{4} \mathrm{C} / \mathrm{Mo} / \mathrm{Si}$. Enfin, nous avons réalisé des multicouches $\mathrm{Sc} / \mathrm{Si}$ qui possède un fort pouvoir réflecteur (supérieur à 35\%) autour de $45 \mathrm{~nm}$. L'ajout de couches barrières dans ces multicouches permet d'améliorer la stabilitié thermique tout en conservant une réflectivité élevée.

\section{Remerciements}

Les dépôts de multicouches ont été réalisés grâce à la Centrale d'Elaboration et de Métrologie d'Optiques X (nommée CEMOX) implémentée par PraXO (Pôle d'optique des Rayons X d'Orsay). Nous remercions tout particulièrement Mourad Idir, Marc Roulliay et Philippe Zeitoun pour leur implication dans ce projet.

Les mesures de réflectivité ont été réalisées au synchrotron ELLETRA sur la ligne BEAR. Nous tenons à remercier tout particulièrement Angelo Giglia, Stefano Nannarone et toute l'équipe de la ligne pour leur aide et leur soutien.

Ce travail a été partiellement financé par le Centre National de la Recherche Scientifique par le programme "Nouveaux matériaux - fonctionnalités nouvelles".

\section{Bibliographie}

[1] M.F. Ravet et al., dans Advances in Optical Thin Films, Claude Amra, Norbert Kaiser, H. Angus Macleod, Eds., Proc. SPIE 5250, 99-108 (2004). 
[2] F. Delmotte et al., J. Phys.IV France 108, 255-258 (2003).

[3] D.L. Windt et al., dans Optics for EUV, X-Ray, and Gamma-Ray Astronomy, Oberto Citterio, Stephen L. O’Dell, Eds., Proc. SPIE 5168, 1-11 (2004).

[4] J.I. Larruquert, J. Opt. Soc. Am. 19, 391-397 (2002).

[5] F. Bridou, B. Pardo, J. Optics (Paris) 21, 183-191 (1990).

[6] Y.A. Uspenskii et al., Opt. Lett. 23, 771 (1998).

[7] A.V. Vinogradov et al., dans Soft X-Ray Lasers and Applications IV, Ernst E. Fill, Jorge J.G. Rocca, Eds, Proc. SPIE 4505, pp. 230-235 (2001).

[8] S.A. Yulin et al., dans Advances in Mirror Technology for X-Ray, EUV Lithography, Laser, and Other Applications, Ali M. Khounsary, Udo Dinger, Kazuya Ota, Eds, Proc. SPIE 5193, pp. 155-163 (2004). 